\title{
THE ROLE OF NON-GAUSSIAN SOURCES IN THE TRANSIENT DYNAMICS OF LONG JOSEPHSON JUNCTIONS*
}

\author{
Claudio Guarcello ${ }^{\dagger}$, Davide Valenti ${ }^{\ddagger}$, Giuseppe Augello \\ BERNARDo SPAGNOLO ${ }^{\S}$
}

\begin{abstract}
Dipartimento di Fisica e Chimica, Group of Interdisciplinary Physics Università di Palermo and CNISM - Unità di Palermo Viale delle Scienze, Edificio 18, 90128 Palermo, Italy
\end{abstract}

(Received May 27, 2013)

We analyze the effects of different non-Gaussian noise sources on the transient dynamics of an overdamped long Josephson junction. We find nonmonotonic behavior of the mean escape time as a function of the noise intensity and frequency of the external driving signal for all the noise sources investigated.

DOI:10.5506/APhysPolB.44.997

PACS numbers: 05.10.-a, 05.40.-a, 05.40.Fb, 85.25.Cp

\section{Introduction}

The advances in the manufacture of high temperature superconductors lead to increase the number of applications connected with Josephson junctions (JJs). Recently, for example, in quantum computing applications, several superconducting qubit circuits based on JJs devices have been analyzed and used [1]. The application fields of the JJs are so numerous and varied that a deep understanding of these devices is essential. Systems such as the ultrafast rapid single-flux-quantum (RSFQ) digital circuits, based on JJs, are strongly influenced by the external environmental variables [2]. These systems show loss of stability, that is switching from the superconducting state to the resistive one, not only due to the thermal noise, but also because

* Presented at the XXV Marian Smoluchowski Symposium on Statistical Physics, "Fluctuation Relations in Nonequilibrium Regime", Kraków, Poland, September 10-13, 2012.

$\dagger$ claudio.guarcello@unipa.it

\$ davide.valenti@unipa.it

$\S$ bernardo.spagnolo@unipa.it 
of distributed non-Gaussian fluctuations. In particular, experimental works indicate the presence of non-thermal fluctuations affecting the dynamics of the bias current in JJs [3]. Recently, many efforts have been performed to study the transient dynamics in short [4, 5] and long [6, 7] JJs in the presence of white and correlated noise sources. The effects of non-Gaussian noise have been studied only in point Josephson junctions [8].

In this work, we analyze the effects of non-Gaussian fluctuations on the dynamics of Josephson junctions. In particular, we analyze how the Lévy noise sources affect the life time of the superconducting state, in the presence of an oscillating driving signal. We observe the appearance of counterintuitive effects due to the noise presence, and suggest how the performance of these circuits can be increased by controlling some external parameters.

\section{The model}

The phase difference of the wave functions in the ground state between left and right superconductive sides of the long Josephson junction, in the overlap geometry, obeys the following stochastic sine-Gordon equation [7, 9]

$$
\bar{\beta} \frac{\partial^{2} \varphi(x, t)}{\partial t^{2}}+\frac{\partial \varphi(x, t)}{\partial t}-\frac{\partial^{2} \varphi(x, t)}{\partial x^{2}}=i(x)-\sin (\varphi(x, t))+i_{\mathrm{f}}(x, t),
$$

with boundary conditions

$$
\frac{\partial \varphi(0, t)}{\partial x}=\frac{\partial \varphi(L, t)}{\partial x}=\Gamma .
$$

Equation (1) is written according to the resistive McCumber-Stewart model framework [9]. Specifically, $\bar{\beta}=1 / \bar{\alpha}^{2}$ is the McCumber-Stewart parameter with the damping coefficient $\bar{\alpha}=\omega_{\mathrm{p}} / \omega_{\mathrm{c}}$, where $\omega_{\mathrm{c}}$ and $\omega_{\mathrm{p}}$ are the characteristic and plasma junction frequencies, respectively. We consider the case with large damping, setting $\bar{\beta} \ll 1$. Furthermore, the space and time variables are normalized to the Josephson length $\lambda_{\mathrm{j}}$ and inverse of $\omega_{\mathrm{c}}^{-1}$, respectively [7]. The two current terms $i(x)$ and $i_{\mathrm{f}}(x, t)$ represent the bias current density and fluctuational current density, both normalized to the critical current density $i_{\mathrm{c}}$ of the junction. Looking at Eq. (2), $L$ is the junction length normalized to $\lambda_{\mathrm{j}}$ and $\Gamma$ is the normalized magnetic field. Here, we consider only the situation with $\Gamma=0$. We analyze only the situation with the bias current density homogeneously distributed along the whole length of the junction, i.e. $i(x)=i_{0}$.

The dynamics of this system is that of a phase string that rolls down along the following tilted dimensionless washboard potential

$$
U(\varphi, x, t)=1-\cos (\varphi(x, t))-i(t) \varphi(x, t),
$$


where

$$
i(t)=i_{0}+A \sin (\omega t),
$$

and $i_{0}=i_{b} / i_{c}, A=i_{s} / i_{c}$ are the normalized dimensionless bias current and driving current amplitude, respectively.

To avoid the creation of fluxons along the junction in the initial time, we impose that the string is entirely located inside one of the potential wells, described by the equations $\varphi(x, 0)=\varphi_{0}=\arcsin \left(i_{0}\right)+2 k \pi$, with $k$ integer number. For simplicity, in our calculations we set $k=0$. The closest left and right maxima, along the washboard potential, are chosen as thresholds for the escape events of the phase cells of the string. The bias current gives rise to a tilted potential. We work with two different bias current values, $i_{0}=0.5$ and $i_{0}=0.9$. In these conditions each well, during the whole dynamics and along the washboard potential, plays the role of a metastable state, with respect to the well located in its right side. Two-dimensional projections of the potential are shown in Fig. 1, for the two values of bias current considered and at three different times. The variations in the slope of the washboard profile and height of the potential barriers, and the absence of structure with peaks and valleys for large values of the driving current are well visible in Fig. 1.
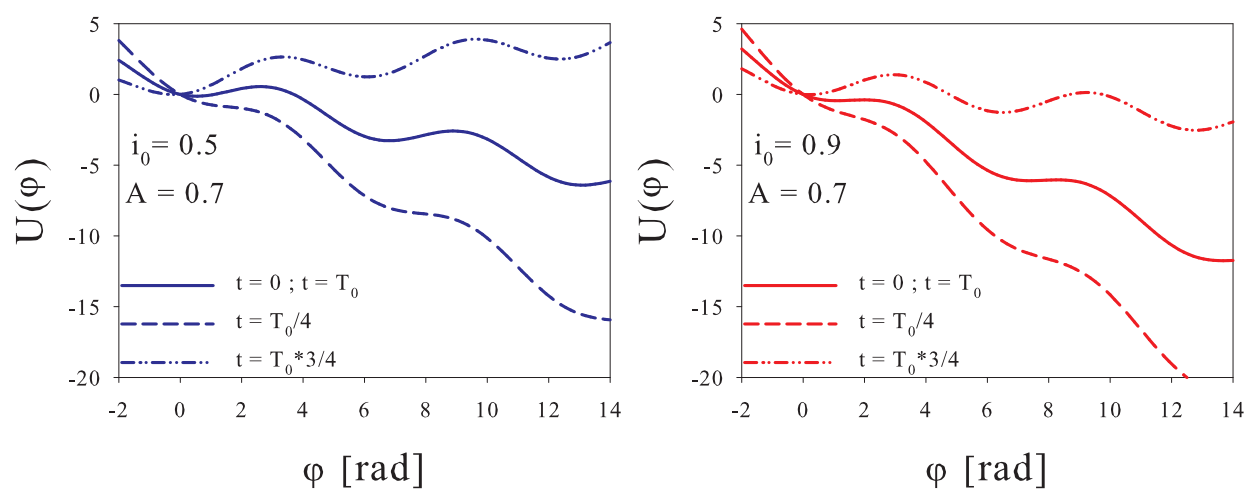

Fig. 1. Washboard potential (3) for a Josephson junction with oscillating driving current (see Eq. (4)) with two different values of bias current, $i_{0}=0.5$ (left panel) and $i_{0}=0.9$ (right panel). Each picture shows three different curves corresponding to three different times: $t=0, t=\frac{T_{0}}{4}$ (maximum negative slope) and $t=\frac{3 T_{0}}{4}$ (minimum slope).

Due to the presence of fluctuations, after some random time, the string overpasses the potential barrier, reaching another washboard minimum. A key quantity for our investigation is the mean escape time (MET), obtained as the average of the random passage times calculated in $N$ different 
numerical realizations. This is the mean permanence time of the total phase string inside the first valley of the washboard potential, that is inside the interval $[-\pi,+\pi]$, according to the nonlinear relaxation time (NLRT) definition [10]. Unlike the mean first passage time, which provides only the average time of the first passage through the right or left potential barrier, the NLRT allows to take into account all the possible retrapping events in the first washboard valley.

\subsection{Lévy noise}

Here, we analyze some properties of the Lévy distributions, which are exploited in this paper to model non-Gaussian perturbations. We recall that Lévy distributions are frequently used to describe the noise impulsive properties in real applications [11]. In a lot of different fields, random fluctuations present jumps or flights, which are impossible to be described by using only a Gaussian noise source [12], and whose evidence can be found in several contexts such as diffusion phenomena by geophysical turbulence, paleoclimatic data, thermohaline circulation in deep ocean, biomedical applications, financial markets, and social systems (for a recent review on Lévy flights, see Ref. [13]).

The probability distributions associated with Lévy processes belong to the class of infinitely divisible distributions, a subset of which is that of stable distributions [14]. These form a family of continuous probability distributions, dependent on two shape parameters $\alpha$ and $\beta$, a scale term $\sigma$ and a real number $\mu$. The parameter $\alpha \in] 0,2]$, known as stability index or characteristic exponent, indicates the asymptotic behavior of the distribution. In fact, for large values of the argument $x$, the distribution tails show an asymptotic power law behavior $\sim|x|^{-(1+\alpha)}$, strongly related to the divergent trend of the moments $\left\langle x^{n}\right\rangle$ for $n \geq 2$ and $\alpha<2$. The index $\beta(\in[-1,1])$ is an asymmetry parameter. In particular, for $\beta=0$, the symmetrical Lévy distributions are obtained. The scale term $\sigma$ is any positive real number that identifies the distribution weight and $\mu$ is any real number indicating, for $\alpha>1$ and $\beta=0$, the average value of the distribution [8].

An $\alpha$-stable (or Lévy) distribution is denoted by $S_{\alpha}(\sigma, \beta, \mu)$ and, whenever $\mu=0$ and $\sigma=1$, the distribution is called standard. In correspondence of $(\alpha, \beta)$ values equal to $(2,0),(1,0),\left(\frac{1}{2}, 1\right)$ and $\left(\frac{1}{2},-1\right)$, one obtains Gaussian (G), Cauchy-Lorentz (CL), Lévy-Smirnov (LS) and reflected (with respect to the vertical axis) Lévy-Smirnov distributions. In our analysis, Lévy noise has been implemented by using a numerical method proposed by Weron [15]. The stochastic dynamics has been obtained integrating Eq. (1) by an implicit finite difference method based on a tridiagonal algorithm. 


\section{Discussions and results}

Our analysis is performed in overdamped regime, setting in Eq. (1) $\bar{\beta}=0.01$. Each MET value is calculated performing the average of the escape time over $N=5000$ experiments for each combination of system parameters. The amplitude of the driving signal is kept fixed, $A=0.7$. This value allows, at least for one of the two $i_{0}$ values chosen, to switch alternatively between superconducting $(i(t)<1)$ and resistive regime $(i(t)>1)$. The values used for the discretized spatial and time steps are $\Delta x=0.05$ and $\Delta t=0.05$, respectively. The curves obtained by numerical calculations are shown in Figs. 2 and 3. Panels (a), (b), (c) of Fig. 2 show results obtained fixing the value of the noise intensity, i.e. $\gamma=0.1$, and varying the driving frequency in the range $\omega \in\left[10^{-2}, 10^{1}\right]$. Each graph contains the curves obtained for a different statistics (depending on $\alpha$ and $\beta$ ) of the noise source, and varying the value of $i_{0}$ and $L$.

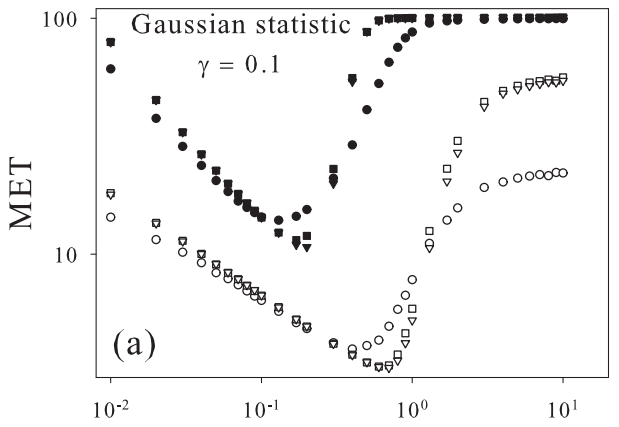

$$
\begin{array}{lll}
\mathrm{L}=1 & , i_{0}=0.5 & \bullet \\
\mathrm{L}=1 & , i_{0}=0.9 & \bigcirc \\
\mathrm{L}=6 & , i_{0}=0.5 & \boldsymbol{\square} \\
\mathrm{L}=6, & , i_{0}=0.9 & \square \\
\mathrm{L}=10, & i_{0}=0.5 & \nabla \\
\mathrm{L}=10, & i_{0}=0.9 & \nabla
\end{array}
$$

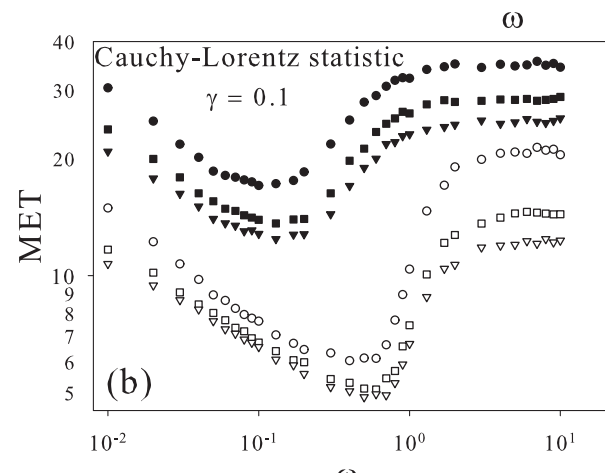

$\omega$

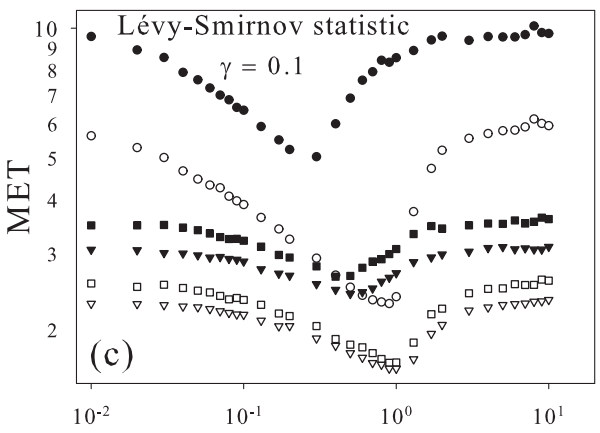

$\omega$

Fig. 2. Log-log plot of the MET as a function of the driving signal frequency for (a) Gaussian, (b) Cauchy-Lorentz (CL) and (c) Lévy-Smirnov (LS) statistics. Each of these pictures shows 6 different curves, obtained for 3 different junction lengths ( $L=1,6,10)$ and 2 values of the bias current $\left(i_{0}=0.5,0.9\right)$. The legend in panel (a) refers also to panels (b) and (c). 
In particular, panels (a), (b), (c) of Fig. 2 show evidence of a nonmonotonic behavior, with a reduction of the lifetime of the metastable state and the appearance of an evident minimum, which is the signature of the resonant activation phenomenon [10, 16, 17]. Moreover, noise sources with Cauchy-Lorentz and Lévy-Smirnov statistics provide further reductions of the MET values, in comparison with results obtained in the presence of Gaussian noise. This stronger decrease of the MET depends on the heavy tails, which characterize Cauchy-Lorentz and Lévy-Smirnov distributions and cause the well known jumps, called Lévy flights [13], affecting the dynamics of the order parameter. The behaviors obtained with $\mathrm{G}$ and CL distributions do not present pronounced variations in the MET trends as the junction length is varied. However, much more important effects appear when the current value switches between $i_{0}=0.5$ and $i_{0}=0.9$. Increasing the value of $i_{0}$, determines a reduction of the MET, due to the enhancement of the potential slope and the consequent lowering of the potential barrier. We can also note a significant shift of the minimum towards higher values of frequency, for bias current going from $i_{0}=0.5$ to $i_{0}=0.9$.

The behavior of MET, as a function of the noise intensity $\gamma$, is shown in Figs. 3 (a)-(c) for a fixed value of frequency, i.e. $\omega=0.4$, and three different values of junction length $(L=1,6,10)$. Each panel presents six curves obtained varying the statistics of the noise source (G, CL, LS distributions) and the bias current $\left(i_{0}=0.5,0.9\right)$. In all curves, the nonmonotonic behavior with maxima reveals the presence of a trapping phenomenon observed for suitable values of the noise intensity, i.e. the noise enhanced stability (NES) phenomenon $[10,18]$. This causes the system to remain in the initial metastable state for a longer time respect to the deterministic case. First, we focus on the results obtained for the higher potential slope, that is $i_{0}=0.9$. In the presence of a Gaussian noise source, two peaks can be clearly distinguished at values of $\gamma$ which differ from each other approximately by two orders of magnitude. The first maximum is less evident, and its amplitude is reduced as the junction length increases. The appearance of this peak is connected with a resonance effect occurring between noise and driving signal. In fact, because of the combined action of the stochastic force and oscillating potential profile, the cells of the phase string tend to remain inside the first valley, causing a resonance trapping phenomenon. The presence of the second peak is instead, due to a further entrapment phenomenon, caused by the return of the phase string within the interval $[-\pi,+\pi]$. This behavior is typically found when the NLRT is calculated, because no absorbing barriers are present. The results obtained using Cauchy-Lorentz noise sources show an evident, but single, peak. The mechanism which determines this maximum is the same as that responsible for the first peak observed in the presence of Gaussian noise. We note, however, a shift towards higher $\gamma$ values, due 
to the limited space displacement [8] that characterizes the Cauchy-Lorentz distributions. The absence of the second peak is instead due to a dynamics mainly governed by intense fluctuations (due to "fat" distribution tails), whose influence grows up as the noise intensity increases. The curves with $i_{0}=0.5$ show the same qualitative behavior as that found for $i_{0}=0.9$, providing a magnification of the same effects. In particular, for $i_{0}=0.5$, the potential barriers are higher and, as a consequence, the MET values are larger than those observed setting $i_{0}=0.9$. Finally we note that, for low values of $\gamma$, the stochastic fluctuations are not able to determine an escape event, so that the phase string remains definitively trapped inside the first washboard valley. In these conditions, the MET reaches approximately the value 100 (see Figs. 3 (a)-(c)), that is the maximum time fixed in our numerical analysis. This maximum time is large enough to allow to consider the string "definitively trapped".
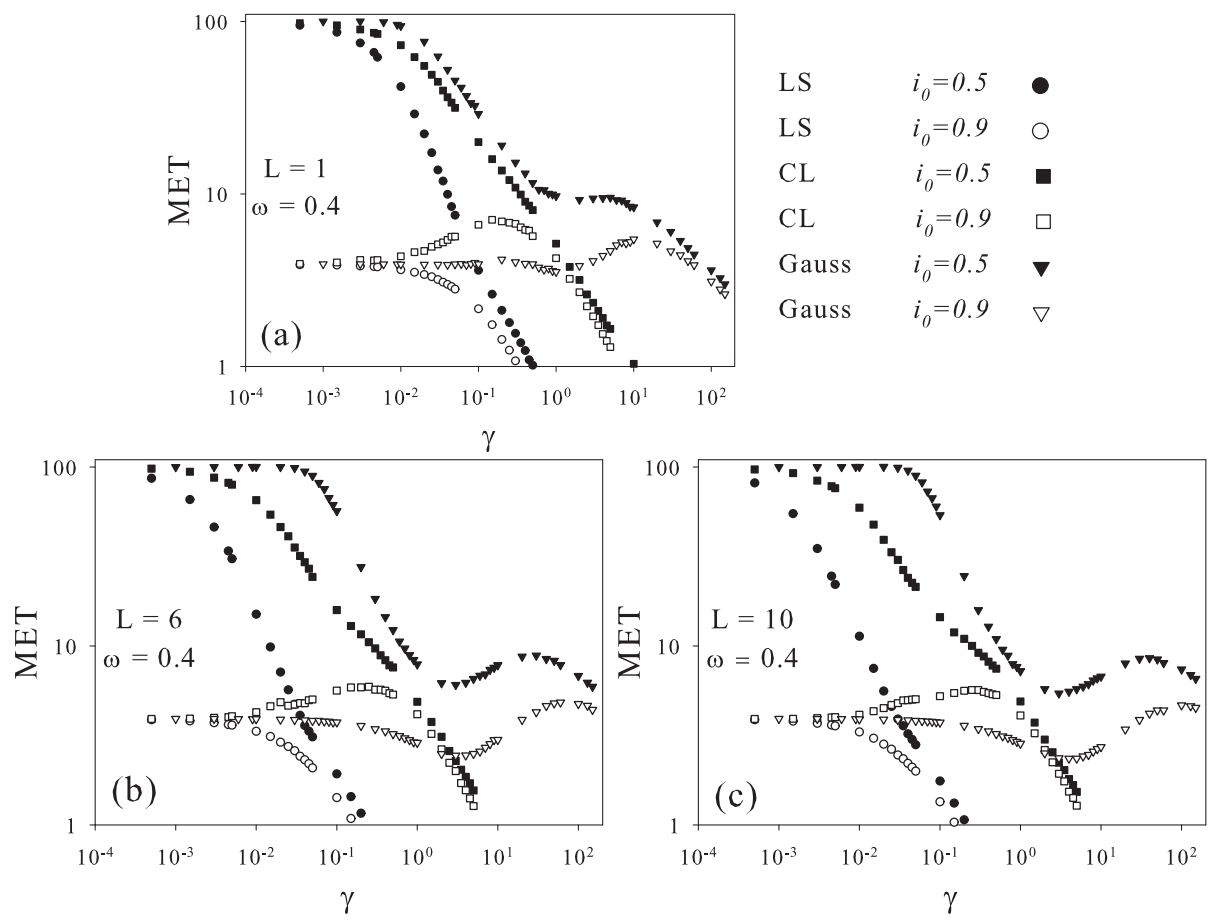

Fig. 3. Log-log plot of the MET as a function of the noise amplitude for three different noise distributions, i.e. Gaussian, Cauchy-Lorentz (CL) and Lévy-Smirnov (LS) statistics. The values of the parameters are $i_{0}=0.5,0.9, \omega=0.4$. The junction length is $L=1$ (panel a), $L=6$ (panel b) and $L=10$ (panel c). The legend in panel (a) refers also to panels (b) and (c). 


\section{Conclusions}

In this paper, we studied the effects of both Gaussian and non-Gaussian noise sources on the lifetime of the superconductive state of a long overdamped Josephson junction, considered in the overlap geometry configu-

ration. Specifically our analysis, performed in the presence of an oscillating driving signal and for three different noise distributions, i.e. Gaussian, Cauchy-Lorentz and Lévy-Smirnov statistics, shows nonmonotonic behavior of the mean escape time, from the superconductive state, as a function both of the frequency $\omega$ of the driving signal and the noise amplitude $\gamma$. These findings clearly indicate the presence of noise induced phenomena such as resonant activation and noise enhanced stability. Moreover, strong differences are observed in the behavior of the lifetime of the superconductive state, depending on the statistics used to obtain the noise source and the values fixed for the bias current and junction length.

\section{REFERENCES}

[1] J. Clarke, F. Wilhelm, Nature (London) 453, 1031 (2008).

[2] A.L. Pankratov, B. Spagnolo, Phys. Rev. Lett. 93, 177001 (2004).

[3] J.T. Peltonen et al., Physica E 40, 111 (2007).

[4] G. Augello, D. Valenti, B. Spagnolo, Int. J. Quan. Info. 6, 801 (2008).

[5] A.V. Gordeeva, A.L. Pankratov, B. Spagnolo, Int. J. Bifur. Chaos 18, 2825 (2008).

[6] G. Augello, D. Valenti, A.L. Pankratov, B. Spagnolo, Eur. Phys. J. B70, 145 (2009).

[7] K.G. Fedorov, A.L. Pankratov, B. Spagnolo, Int. J. Bifur. Chaos 18, 2857 (2008).

[8] G. Augello, D. Valenti, B. Spagnolo, Eur. Phys. J. B78, 225 (2010).

[9] A. Barone, G. Paternò, Physics and Applications of the Josephson Effect, Wiley, New York 1982.

[10] A.A. Dubkov, N.V. Agudov, B. Spagnolo, Phys. Rev. E69, 061103 (2004).

[11] W. Szajnowski, J. Wynne, IEEE Signal Process. Lett. 8, 151 (2001).

[12] W.A. Woyczynski, In Lévy Processes: Theory and Applications, O.E. Barndorff-Nielsen, T. Mikosch, S.I. Resnick (Eds.), p. 241, Birkhäuser, Boston 2001; A.A. Dubkov, B. Spagnolo, Acta Phys. Pol. B 38, 1745 (2007); A.A. Dubkov, A. La Cognata, B. Spagnolo, J. Stat. Mech.: Theory Exp. 1, P01002 (2009); A.A. Dubkov, B. Spagnolo, Eur. Phys. J. B65, 361 (2008).

[13] A.A. Dubkov, B. Spagnolo, V.V. Uchaikin, Int. J. Bifur. Chaos 18, 2649 (2008).

[14] W. Feller, An Introduction to Probability Theory and Its Applications, Vol. 2, John Wiley Sons, Inc., New York 1971. 
The Role of Non-Gaussian Sources in the Transient Dynamics of Long ... 1005

[15] A. Weron, Stat. Prob. Lett. 28, 165 (1996).

[16] R. Doering, J.C. Gadoua, Phys. Rev. Lett. 69, 2318 (1992).

[17] N. Mantegna, B. Spagnolo, Phys. Rev. Lett. 84, 3025 (2000); A. Fiasconaro, B. Spagnolo, Phys. Rev. E83, 041122 (2011).

[18] N.V. Agudov, B. Spagnolo, Phys. Rev. E64, 035102 (2001). 